OPEN ACCESS

Edited by:

Gislane Lelis Vilela de Oliveira,

São Paulo State University, Brazil

Reviewed by:

Paul De Vos,

University Medical Center Groningen,

Netherlands

Zhengxiang $\mathrm{He}$,

Icahn School of Medicine at Mount

Sinai, United States

${ }^{*}$ Correspondence:

Allison K. Ehrlich

akehrlich@ucdavis.edu

${ }^{\dagger}$ These authors have contributed equally to this work

Specialty section:

This article was submitted to

Mucosal Immunity,

a section of the journal

Frontiers in Immunology

Received: 14 September 2020

Accepted: 23 November 2020

Published: 21 January 2021

Citation:

Kahalehili HM, Newman NK

Pennington JM, Kolluri SK,

Kerkvliet NI, Shulzhenko N, Morgun A and Ehrlich AK (2021) Dietary Indole-3-

Carbinol Activates AhR in the Gut,

Alters Th17-Microbe Interactions, and

Exacerbates Insulitis in NOD Mice.

Front. Immunol. 11:606441.

doi: 10.3389/fimmu.2020.606441

\section{Dietary Indole-3-Carbinol Activates AhR in the Gut, Alters Th17-Microbe Interactions, and Exacerbates Insulitis in NOD Mice}

\author{
Heather M. Kahalehili ${ }^{1 \dagger}$, Nolan K. Newman ${ }^{2 \dagger}$, Jamie M. Pennington ${ }^{3}$, Siva K. Kolluri ${ }^{3}$, \\ Nancy I. Kerkvliet ${ }^{3}$, Natalia Shulzhenko ${ }^{4}$, Andrey Morgun ${ }^{2}$ and Allison K. Ehrlich ${ }^{1 *}$ \\ ${ }^{1}$ Department of Environmental Toxicology, University of California, Davis, CA, United States, ${ }^{2}$ College of Pharmacy, Oregon \\ State University, Corvallis, OR, United States, ${ }^{3}$ Department of Environmental and Molecular Toxicology, Oregon State University, \\ Corvallis, OR, United States, ${ }^{4}$ Department of Biomedical Sciences, Oregon State University, Corvallis, OR, United States
}

The diet represents one environmental risk factor controlling the progression of type 1 diabetes (T1D) in genetically susceptible individuals. Consequently, understanding which specific nutritional components promote or prevent the development of disease could be used to make dietary recommendations in prediabetic individuals. In the current study, we hypothesized that the immunoregulatory phytochemcial, indole-3-carbinol (I3C) which is found in cruciferous vegetables, will regulate the progression of T1D in nonobese diabetic (NOD) mice. During digestion, I3C is metabolized into ligands for the aryl hydrocarbon receptor (AhR), a transcription factor that when systemically activated prevents T1D. In NOD mice, an I3C-supplemented diet led to strong AhR activation in the small intestine but minimal systemic AhR activity. In the absence of this systemic response, the dietary intervention led to exacerbated insulitis. Consistent with the compartmentalization of AhR activation, dietary I3C did not alter T helper cell differentiation in the spleen or pancreatic draining lymph nodes. Instead, dietary $13 \mathrm{C}$ increased the percentage of CD4 ${ }^{+}$ROR $\gamma^{+}{ }^{+}$Foxp3 $^{-}$(Th17 cells) in the lamina propria, intraepithelial layer, and Peyer's patches of the small intestine. The immune modulation in the gut was accompanied by alterations to the intestinal microbiome, with changes in bacterial communities observed within one week of I3C supplementation. A transkingdom network was generated to predict host-microbe interactions that were influenced by dietary I3C. Within the phylum Firmicutes, several genera (Intestinimonas, Ruminiclostridium 9, and unclassified Lachnospiraceae) were negatively regulated by I3C. Using AhR knockout mice, we validated that Intestinimonas is negatively regulated by AhR. I3C-mediated microbial dysbiosis was linked to increases in CD25 $5^{\text {high }}$ Th17 cells. Collectively, these data demonstrate that site of AhR activation and subsequent interactions with the host microbiome are important considerations in developing AhR-targeted interventions for T1D.

Keywords: indole-3-carbinol (I3C), type 1 diabetes, aryl hydrocarbon receptor (AhR), Th17 cells (Th17), microbiome 


\section{INTRODUCTION}

Type 1 diabetes (T1D) is characterized by uncontrolled hyperglycemia resulting from autoimmune-mediated destruction of insulin-producing beta cells. Unlike other immune-mediated diseases that can be treated by combatting the underlying inflammatory response, T1D is generally diagnosed after the majority of beta cells have been destroyed. Early interventions targeting risk factors that influence T1D progression could negate the need for life-long insulin replacement (1). In genetically susceptible individuals, the diet is among the external factors known to influence the rate of T1D development $(2,3)$. The influence of diet on T1D is thought to reflect modulation of mucosal immune responses, intestinal permeability, and microbiome diversity (4-7).

One dietary component that has been shown to modulate autoimmune disease development is ligands that activate the aryl hydrocarbon receptor (AhR) (8). AhR modulates many aspects of the autoimmune response and is also becoming known as a major regulator of gut homeostasis (9). Several studies have shown that AhR activation by high affinity ligands suppresses immune-mediated diseases, including T1D (10-14). However, the immunological outcome of dietary AhR ligand intake could be unpredictable due to the heterogeneity of ligands, their AhR affinity, and amount consumed. While a diet rich in AhR ligands would be predicted to mimic the studies showing the therapeutic benefit of high affinity ligands in preventing the progression of T1D, it is possible that consumption of a diet low in AhR ligands could contribute to T1D progression. This concern arises based on our recent studies demonstrating that a low level of AhR activation can lead to the differentiation of Th17 cells instead of Tregs (15), which could promote, rather than prevent T1D progression (16-19).

Indole-3-carbinol (I3C), is a dietary AhR ligand precursor formed as a hydrolysis product of glucobrassicin, a compound found in cruciferous vegetables such as broccoli, kale, and brussel sprouts. I3C is further broken down by the acidic environment in the stomach into dimers, trimers and tetramers, some of which have high affinity for the AhR (20). Intake of I3C has been estimated at $0.1-1.6 \mathrm{mg} / \mathrm{kg}$ (21); I3C intake would be lower in individuals who avoid cruciferous vegetables, and higher in those who consume a cruciferous rich diet or who take widely available I3C supplements. The impact of dietary I3C on the inflammatory response has been studied in several C57BL/6 murine models including DSS-induced colitis $(22,23)$, Citrobacter rodentiuminduced intestinal inflammation [ (24), Clostridium difficile infection (25), and food allergy (26)]. Even with the varying I3C doses used in these studies (ranging from 150-2,000 ppm), I3C supplementation activated $\mathrm{AhR}$ and reduced the associated immunopathology. In addition to direct effects on the intestinal immune response, supplementation of the diet with I3C also strongly shifts the gut microbiome composition $(22,23,27)$. Although dietary I3C led to activation of AhR in each study, the specific changes in microbiota diversity varied between studies and occurred by both AhR-dependent and independent mechanisms. Thus, I3C alters gut health through two interacting pathways, by modulating the immune response and the microbiota.

Since both of these pathways can influence T1D progression $(5,28)$, in the present study we use the non-obese diabetic (NOD) mouse model of autoimmune diabetes to study the effects of dietary supplementation with I3C on gut immunemicrobe interactions, and subsequent development of insulitis.

\section{MATERIAL AND METHODS}

\section{Animals}

NOD/ShiLtJ (NOD) mice were obtained from The Jackson Laboratory and maintained in the specific pathogen-free animal facility at Oregon State University. NOD.AhR-/- mice were generated by backcrossing B6.129-AHRtm1Bra/J onto the NOD/LtJ background for more than 13 generations. All experiments used female littermate-matched mice. All animal procedures were carried out following protocols approved by the Institutional Animal Care and Use Committee at Oregon State University.

\section{Preliminary Oral Gavage Studies}

Cl-BBQ (11-chloro-7H-benzimidazo[2,1-a]benzo[de]Isoquinolin-7-one; Chembridge) or I3C (Sigma) were dissolved in DMSO-Cremaphor-Peceol (30\%:20\%:50\%) by sonicating in a 37 degree water bath for $1 \mathrm{~h}$. Female NOD mice were administered $200 \mathrm{ul}$ of Cl-BBQ $(45 \mathrm{mg} / \mathrm{kg})$, I3C $(250 \mathrm{mg} / \mathrm{kg})$ or the vehicle control by oral gavage. Mice were sacrificed at 6 and $24 \mathrm{~h}$ after oral gavage to measure AhR activation.

\section{Dietary I3C}

NOD mice were fed normal chow until 7 weeks of age when they were transitioned to either a synthetic diet (AIN93M; Research Diets) alone or supplemented with 2,000 ppm I3C. Mice were maintained on these diets through the remainder of the study (12 weeks). Food consumption was tracked daily by weighing the remaining food for the first week of the study and then weekly from weeks 8 to 12. Body weight was likewise measured daily for the first week and then once a week for the remainder of the study. Two independent experiments containing three or four individual birth cohorts were conducted.

\section{Real-Time PCR and Cyp1a1 Measurement}

The liver and small intestine were removed from mice and stored in RNAlater for immediate stabilization prior to RNA isolation using RNeasy Mini Kit (Qiagen). cDNA was synthesized using the High-Capacity cDNA Reverse Transcription Kit (Applied Biosystems). qPCR reactions were performed on an Agilent Stratagene Real-Time PCR system (Applied Biosystems) using SYBR Green/ROX Master Mix from SA Biosciences. Cypla1 levels were normalized to Actb using primers from SA Biosciences.

\section{Assessment of Disease Progression}

Insulitis was scored on sequential hematoxylin and eosin (H\&E) stained pancreas sections separated by $200 \mu \mathrm{M}$ with at least 50 
islets scored per pancreas. Islets were scored as no infiltration, less than $50 \%$ infiltration or greater than $50 \%$ infiltration.

\section{Cell Isolation and Flow Cytometry}

At 12 weeks of age, mice were sacrificed, pancreatic lymph node (PLN), spleen, and the small intestine were excised. Single cell suspensions were prepared from the spleen, PLN, and Peyer's Patches by mechanical disruption between frosted slides. Splenoyctes were further processed by hypotonic red blood cell lysis. Isolation of lamina propria (LP) cells and intraepithelial lymphocytes (IEL) from the small intestine was performed as previously described (29). Briefly, Peyer's patches were excised and the epithelial layer of the small intestine was isolated through sequential washes in a $5 \mathrm{mM}$ EDTA and $0.145 \mathrm{mg} / \mathrm{ml}$ DTT solution while stirring at $37^{\circ} \mathrm{C}$. LP cells were isolated from the remaining intestinal tissue by mincing and digesting the tissue using $0.2 \mathrm{U} / \mathrm{ml}$ Liberase TM and $0.05 \%$ DNase while shaking at $37^{\circ} \mathrm{C}$. The digested tissue was washed three times with a $3 \%$ FBS solution and successively filtered after each wash through two 70 $\mu \mathrm{m}$ filters and one $40 \mu \mathrm{m}$ filter in preparation for cell staining.

Cells were stained with fixable viability dye (eBioscience), Fc receptors were blocked with rat IgG (Jackson ImmunoResearch) and the cells were stained with the following antibodies CD45 (30-F11), CD4 (RM4-4), Nrp1 (3DS304M) from eBioscience, Lag3 (C9B7W), CD25 (PC61) and CD210 (1B1.3a), CD8 (536.7) from $\mathrm{BD}$ Biosciences and Tim3 from Biolegend. For intracellular staining, cells were fixed and permeabilized using the Foxp3 Fixation/Permeabilization buffer (eBioscience) and stained with Tbet (4B10) and Foxp3 (FJK168) from eBioscience, and ROR $\gamma$ t (Q31-378) from BD Biosciences. For cytokine staining in splenoyctes, cells were stimulated with PMA, ionomycin, brefeldin $\mathrm{A}$, and monensin (eBioscience) for $4 \mathrm{~h}$ in culture and stained with IFN $\gamma$ (XMG1.2), IL-10 (JES5-16ES), IL22 (1H8PWSR) from eBioscience and IL-17 (TC11-18H10) from BD Biosciences.

Data were acquired on a Cytoflex flow cytometer (Beckman Coulter). Data were compensated and analyzed using FlowJo (Treestar) software. Fluorescence minus one (FMO) controls were used for setting gates for analysis.

\section{S rRNA Gene Sequencing Analysis}

For microbial measurements, fresh stool pellets were collected at 7 , 8 , and 12 weeks of age and immediately stored at $-80^{\circ} \mathrm{C}$. To extract microbial DNA, frozen fecal pellets were resuspended in $1.4 \mathrm{ml}$ ASL buffer (Qiagen) and homogenized with $2.8 \mathrm{~mm}$ ceramic beads followed by $0.5 \mathrm{~mm}$ glass beads using an OMNI Bead Ruptor (OMNI International). DNA was extracted from the entire resulting suspension using QiaAmp mini stool kit (Qiagen) according to manufacturer's protocol. DNA was quantified using Qubit broad range DNA assay (LifeTechnologies).

The primers $319 \mathrm{~F}$ and $806 \mathrm{R}$ were used to amplify the V3-V4 domain of the $16 \mathrm{~S}$ rRNA using a two-step PCR procedure. In step one of the amplification procedure, both forward and reverse primers contained an Illumina tag sequence (bold), a variable length spacer (no spacer, C, TC, or ATC for 319F; no spacer, G, TG, ATG for 806R) to increase diversity and improve the quality of the sequencing run, a linker sequence (italicized), and the 16S target sequence (underlined). In step two, each sample was barcoded with a unique forward and reverse barcode combination using forward primers (AATGATACGG CGACCACCGAGATCTACACNNNNNNNNTCGTCGG CAGCGTC) with an Illumina P5 adapter sequence (bold), a unique $8 \mathrm{nt}$ barcode $(\mathrm{N})$, a partial matching sequence of the forward adapter used in step one (underlined), and reverse primers (CAAGCAGAAGACGGCATACGAGATNNNN NNNNGTCTCGTGGGCTCGG) with an Illumina P7 adapter sequence (bold), unique $8 \mathrm{nt}$ barcode $(\mathrm{N})$, and a partial matching sequence of the reverse adapter used in step one (underlined). The final product was quantified on the Qubit instrument using the Qubit Broad Range DNA kit (Invitrogen) and individual amplicons were pooled in equal concentrations. The pooled library was cleaned utilizing Ampure XP beads (Beckman Coulter) then the band of interest was further subjected to isolation via gel electrophoresis on a $1.5 \%$ Blue Pippin HT gel (Sage Science). The library was quantified via qPCR followed by 300-bp paired-end sequencing using an Illumina MiSeq instrument in the Genome Center DNA Technologies Core, University of California, Davis. The QIIME 2 (30) bioinformatics pipeline (v. 2018.8.0) was used to demultiplex and quality filter the forward-end fastq files. Denoising was performed using DADA2 (31). The raw data can be accessed at NCBI Sequence Read Archive (SRA) (Accession \#PRJNA679964).

\section{Data Pre-Processing}

Host parameters were first normalized by birth cohort. Host parameters that were analyzed in both independent experiments were subsequently normalized across the two studies. For microbial data, after excluding amplicon sequence variant (ASV) singletons, a threshold of $99.5 \%$ cumulative abundance across all samples in both experiments was used to select the most abundant ASVs for downstream analysis. Following this, ASVs were relativized per million and quantile normalized (per experiment), then $\log 2$ transformed for downstream analysis.

\section{Reconstruction of Transkingdom Network}

The network analysis has been performed as described in previous papers $(32,33)$, except for the minor modifications outlined below. Spearman rank correlations were conducted for all experimental groups (Control and I3C for each of the two experiments) between all pairs of ASVs and host parameters. Edges that did not demonstrate the same sign of correlation direction (positive or negative) across all groups and those that were not compliant with causality principles (34) were removed. Similarly, those edges that did not contain nodes consistent in fold change direction across experiments were also removed. The metacor function of the meta package [ $\mathrm{v} 4.12-0$ (35); ] in $\mathrm{R}(\mathrm{v}$ 3.5.1) was used to calculate the fixed effect model p-values based on Fisher's Z-Transformation of proportions of the correlations across the four groups. Prior to calculating FDRs, the correlations between microbiota and phenotypes were first filtered for those that had an individual p-value $<0.6$ across all groups, with the exception of the PP_RORgt+Foxp3$<==>$ ASV21 and IEL_RORgt+Foxp3-<==>ASV290 edges $(\mathrm{FDR}=0.135$ for both $)$. These two additional edges were kept 
in the network despite not passing individual p-value thresholds due to the high biological importance of the ROR $\gamma \mathrm{t}^{+}$Foxp $3^{-}$cell populations. Therefore, we checked for edges connecting to that cell population that barely missed making the network. FDR was then calculated on the correlation $p$-values for each of the following groups: (1) between microbes; (2) between microbes and host parameters; (3) between host parameters. Microbe-host parameter edges with an FDR $<0.15$ were retained in the network. Edges between microbiota were required to have an FDR $<0.05$ while those between host parameters were required to have an FDR $<0.1$.

\section{Network Analysis}

Networks were visualized using Cytoscape v3.7.2 (36). Silva IDs were used in labeling the important microbes (as determined by their high normalized bipartite betweenness centrality [BiBC] and degree). The Python module NetworkX v2.2 was used to calculate $\mathrm{BiBC}$ and degree between groups, as well as to randomly generate the 10,000 networks used in validating the $\mathrm{BiBC}$ and degree results. $\mathrm{BiBC}$ values were calculated as previously described (32), then normalized by the number of nodes in each group. The 10,000 binomial random (ErdosRenyi) networks were generated from the $G(m, n)$ ensemble with $\mathrm{m}=221$ (to match the number of edges in the real network) and $n=109$ (the number of nodes). The 2D contour histogram BiBC-degree distribution was plotted using the online tool Plotly (https://plot.ly/). Probability density was used as a measurement of the likelihood of randomly finding a node with the given $\mathrm{BiBC}$ and degree (or higher). A large value (i.e. a darkcolored space in the contour map) indicates that a node in that area typically occurs in random networks size-matched to the network generated from the data.

\section{Statistical Analysis}

With the exception of the network analyses, statistical analyses were performed using Graphpad Prism. For insulitis and host parameters, data were normalized by birth cohort/littermates. For comparing two groups, a Student's t test was performed. For multiple comparisons, one-way ANOVA with Tukey's test was used. $\mathrm{P}<0.05$ was considered statistically significant. All plotted data points represent an individual mouse.

\section{RESULTS}

\section{Dietary I3C Strongly Activates AhR in the Intestine but Induces Limited Systemic AhR Activation}

Activation of the AhR by I3C supplementation has not been previously studied in NOD mice. To determine the extent by which $\mathrm{I} 3 \mathrm{C}$ can induce $\mathrm{AhR}$ activation, we first measured activation following oral gavage with $\mathrm{I} 3 \mathrm{C}$ as compared to $\mathrm{Cl}-\mathrm{BBQ}$, a high affinity AhR ligand previously shown to suppress T1D in NOD mice $(10,12)$. In C57BL/6 mice, $250 \mathrm{mg} / \mathrm{kg}$ of I3C (or dietary equivalent) is on the higher range of previously reported doses used to activate the $\operatorname{AhR}(22-24,26,27,37)$ [; higher doses $(>500 \mathrm{mg} / \mathrm{kg})$ of I3C are associated with neurotoxicity and increased mortality (20)]. To account for the $A h R^{d}$ allele, which has a $\sim 10$ fold lower sensitivity to AhR ligands $(12,38)$, a dose $250 \mathrm{mg} / \mathrm{kg}$ I3C was chosen to maximize AhR activation and minimize toxicity.

NOD mice were administered I3C by oral gavage and Cyp $1 a 1$ induction was measured in the liver, pancreatic lymph node, and small intestine (duodenum, jejunum, and ileum). AhR response elements upstream of Cypla1 make it a highly sensitive target of $\mathrm{AhR}$ and, as a result, the induction of Cypla1 is a commonly used biomarker for AhR activation (39). The positive control, Cl$\mathrm{BBQ}$, resulted in sustained Cyp1a1 induction in the liver and pancreatic lymph nodes at 6 and $24 \mathrm{~h}$ post-oral gavage. In contrast, I3C only resulted in transient Cyp $1 a 1$ induction, which was 4 - and 20-fold lower in the liver and pancreatic lymph node, respectively, at $6 \mathrm{~h}$ post-oral gavage. By $24 \mathrm{~h}$ after I3C administration, Cypla1 was back to baseline (Figure 1A). Furthermore, I3C did not induce Cypla1 in the duodenum, jejunum, and ileum of the small intestine following oral gavage (Figure 1B).

In contrast to gavage administration, one week of diet supplementation with I3C (2,000 ppm, equivalent to $250 \mathrm{mg} /$ $\mathrm{kg} /$ day based on food consumption) was capable of inducing strong Cypla1 in the small intestine (median of $\sim 1,000$-fold increase; Figure 1C). However, as with oral gavage, AhR activation was likewise limited systemically following dietary exposure. Given the apparent lack of systemic AhR activation following dietary I3C supplementation, it was difficult to predict how it would impact T1D pathogenesis. However, the ability of I3C to induce Cyp1a1 locally in the intestine (Figure 1D), provided an unexpected opportunity to determine how AhR activation in the gut alters the development of T1D.

\section{Dietary I3C Exacerbates Insulitis in Nonobese Diabetic Mice}

To determine if strong AhR activation in the intestine was sufficient to suppress insulitis, NOD mice were fed a diet supplemented with 2,000 ppm I3C from seven to twelve weeks of age, during which time insulitis is known to progress. Initiating the dietary regimen at this timepont was selected to correspond to the treatment timing in our previous studies with Cl-BBQ and TCDD (10). At seven weeks of age, NOD mice were randomized by litter, cage, and weight then assigned to a control or I3C-diet fed group (Figure 2A). Mice fed an I3Csupplemented diet showed no difference in food consumption or body weight compared to control mice (Figures 2B, C). Mice averaged 2-2.5 g of food/week resulting in an average exposure of 190-260 mg/kg/day of I3C.

At 12 weeks of age, mice were euthanized and islet infiltration was scored in the sectioned pancreas. Unexpectedly, mice given the diet supplemented with $\mathrm{I3C}$ had significantly increased insulitis. The increase in insulitis was primarily a reflection of the significant increase in the percentage of islets per pancreas that were heavily infiltrated (50\% infiltration, $\mathrm{p}<0.05)$ in mice fed the I3C-supplemented diet; the percentage of islets with $<50 \%$ infiltration was unchanged between treatment groups. This pattern was observed in two independent experiments (Figure 2D). 

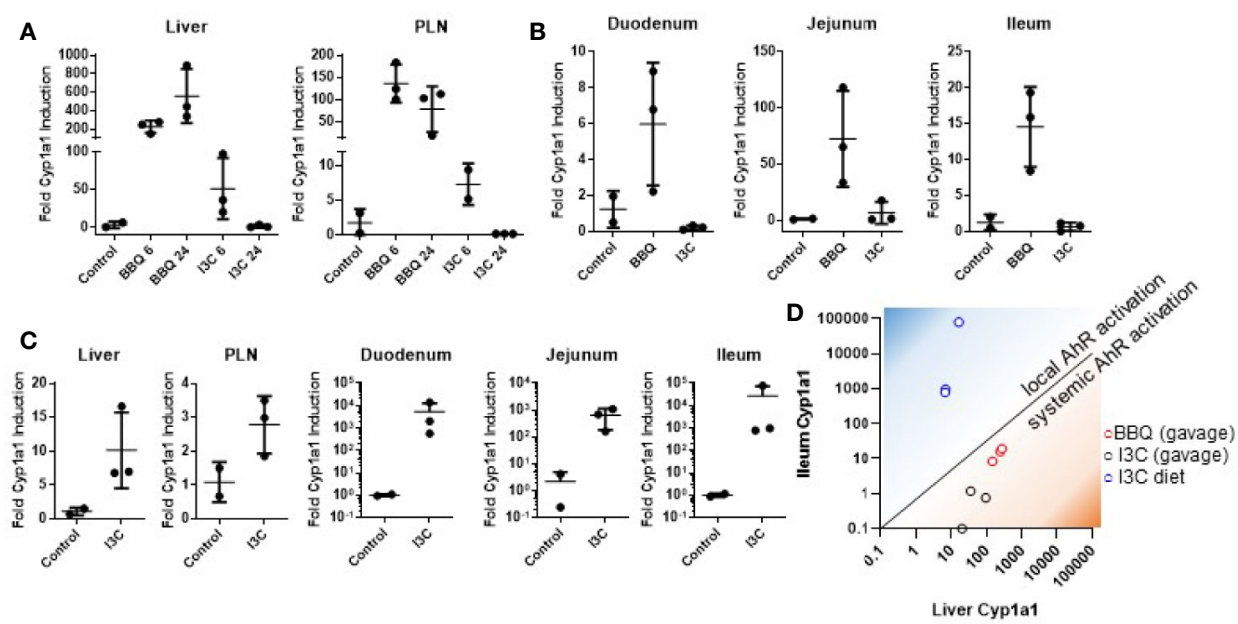

FIGURE 1 | Dietary I3C strongly activates AhR in the intestine but not systemically. Cyp1a1 expression was measured in the liver and pancreatic lymph node of NOD mice following treatment with either $13 \mathrm{C}(250 \mathrm{mg} / \mathrm{kg})$ or Cl-BBQ $(45 \mathrm{mg} / \mathrm{kg})$ at 6 and $24 \mathrm{~h}$ post-oral gavage (A) and in the small intestine $6 \mathrm{~h}$ post-oral gavage (B). (C) Cyp1a1 was analyzed after one week of dietary 13C (2,000 ppm) in the indicated organs. (D) Comparison between local (ileum) and systemic (liver) AhR activation by $\mathrm{Cl}-\mathrm{BBQ}$ (gavage), I3C (gavage), and dietary I3C.

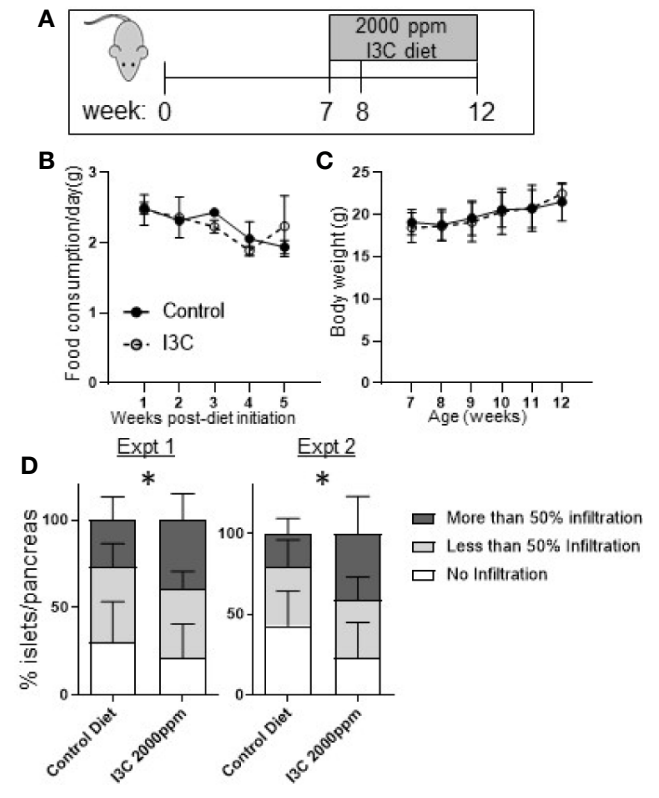

FIGURE 2 | Dietary I3C exacerbates insulitis in NOD mice. (A) Schematic of experimental design. From 7 to 12 weeks of age, NOD mice were maintained on a synthetic AhR ligand-free diet or diet supplemented with 2,000 ppm I3C. Stool samples were collected at 7, 8, and 12 weeks of age (at the time of sacrifice). Food consumption (B) and body weight (C) were measured weekly. (D) In two independent experiments, insulitis was scored as no infiltration, less than $50 \%$, and greater than $50 \%$ of islets infiltrated. Data are presented as the average from each mouse per group. Experiment $1: n=9$ mice/group; Experiment 2: $n=7-8$ mice/group. ${ }^{*} p<0.05$.

\section{Dietary I3C Increases Th17 Cells in the Intestine}

Th17 cells and Tregs (Foxp $3^{+}$and $\operatorname{Tr} 1$ ) are targets of AhR activation and implicated in promoting and inhibiting the autoimmune destruction of beta cells, respectively $(16,18,19,40-42)$. Therefore, alterations in markers representing $\mathrm{CD}^{+} \mathrm{T}$ cell helper subsets were examined in the spleen, pancreatic lymph node, Peyer's patches, the small intestine lamina propria, and the small intestine intraepithelial cells. Significant increases were found in $\mathrm{CD}^{+}{ }^{+} \mathrm{Foxp} 3$ RORyt ${ }^{+}$Th17 cells in the lamina propria (2.3-fold increase), intraepithelial layer (2.7-fold increase), and Peyer's Patches (1.6fold increase; Figures 3A-D). A subset of lamina propria $\mathrm{CD} 4^{+}$Foxp $3^{-}$cells expressing high levels of ROR $\gamma t$ expressed the T cell activation marker $\mathrm{CD} 25^{+}$and were significantly increased as well (1.7-fold increase; Figure 3E). Confirming that dietary I3C increased Th17 cells, LPL cells from a subset of mice were stimulated with PMA/ionomycin and IL-17 was measured by flow cytometry (Figure 3F). No significant changes in $\operatorname{Tr} 1$ cells, Th1 cells, and Th17 cells were found in the spleen or pancreatic lymph nodes, although a trend toward increased Th17 cells was observed in the PLN (Supplementary Figure 1), consistent with the minimal AhR activation at these sites. While the majority of significant changes in immune cell markers were identified in the intestine, some minor yet significant populations were changed in the spleen. Significant changes in all analyzed populations are listed in Figure 3G.

\section{Dietary I3C Alters the Gut Microbiome}

Since modulation of immune cell populations in the gut by dietary $\mathrm{I} 3 \mathrm{C}$ can arise from AhR-mediated changes in gut 

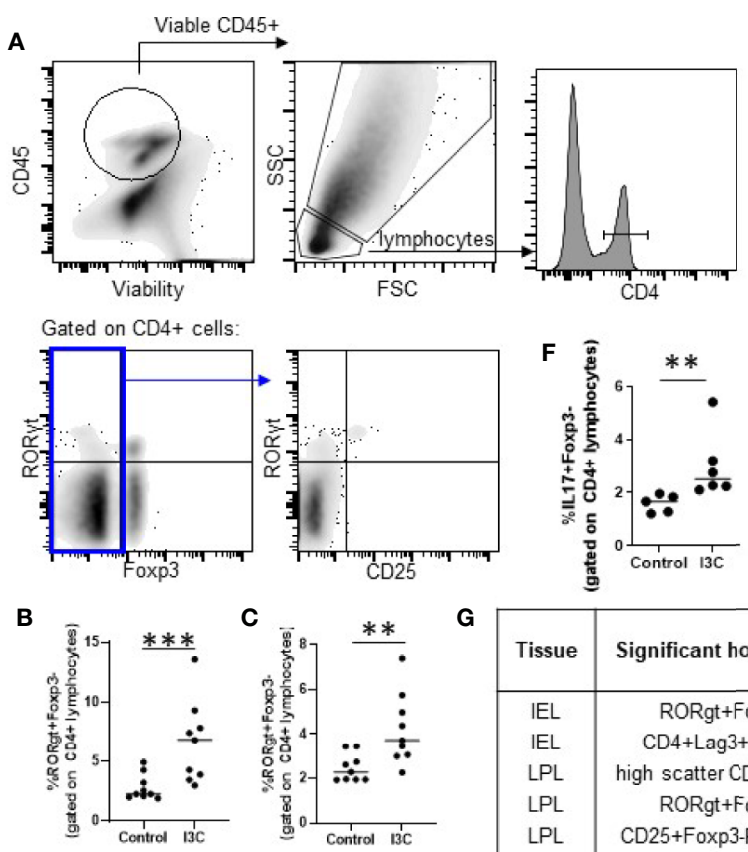

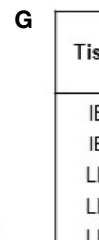
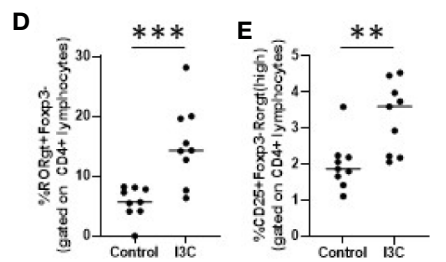

\begin{tabular}{|c|c|c|c|c|c|}
\hline Tissue & Significant host factors & $\begin{array}{c}\text { Control } \\
\text { (avg) }\end{array}$ & $\begin{array}{c}13 \mathrm{C} \\
\text { (avg) }\end{array}$ & $\begin{array}{l}\text { Ratio } \\
\text { (I3C: } \\
\text { Control) }\end{array}$ & $\begin{array}{c}\mathbf{p} \\
\text { value }\end{array}$ \\
\hline $\mathrm{IEL}$ & RORgt+Foxp3- & 5.64 & 15.38 & 2.73 & 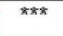 \\
\hline $\mathrm{IEL}$ & CD4+Lag3+Foxp3- & 3.83 & 8.48 & 2.22 & ** \\
\hline LPL & high scatter CD4-IL10R+ & 36.36 & 24.86 & 0.68 & 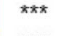 \\
\hline LPL & RORgt+Foxp3- & 2.85 & 6.63 & 2.33 & $\times x \times$ \\
\hline LPL & CD25+Foxp3-RORgthigh & 1.99 & 3.30 & 1.66 & ** \\
\hline $\mathrm{PP}$ & Nrp1-Foxp3+ & 6.43 & 8.00 & 1.24 & $\star \star \star$ \\
\hline $\mathrm{PP}$ & RORgt+Foxp3- & 2.48 & 4.22 & 1.71 & ** \\
\hline $\mathrm{PP}$ & RORgt+Foxp3+ & 1.38 & 2.05 & 1.48 & * \\
\hline Spleen & CD4+IL-22+Foxp3- & 0.64 & 0.87 & 1.37 & ** \\
\hline Spleen & CD4+Nrp1highFoxp3- & 2.54 & 2.13 & 0.84 & * \\
\hline Spleen & $\mathrm{CD} 4+\mathrm{CD} 25+\mathrm{Foxp} 3-$ & 2.00 & 1.56 & 0.78 & * \\
\hline Pancreas & Insulitis $(>50)$ & 26.56 & 39.08 & 1.47 & * \\
\hline Ileum & Ileum Cyp1a1 & 1.88 & 439.77 & 233.92 & 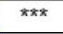 \\
\hline
\end{tabular}

FIGURE 3 | Dietary I3C increases Th17 cells in the intestine. Representative gating strategy (A) and population frequency of Th17 cells in the small intestine lamina propria (B, E), Peyer's patches (C), and intraepithelial layer (D). A subset of lamina propria cells were stimulated with PMA/ionomycin and stained for IL-17 (F). Table of significant changes in all analyzed populations (G). ${ }^{\star} p<0.05 ;{ }^{* \star} p<0.01 ;{ }^{\star \star \star} p<0.001$.

microbiota diversity, we examined whether dietary I3C altered gut microbial composition in NOD mice. Stool samples were collected prior to the dietary intervention at 7 weeks of age, after one week of the diet, and at the time of sacrifice at 12 weeks of age.

Within one week of starting the new diets, there was a strong shift in the microbial communities of both groups of mice fed the synthetic control diet or the I3C-supplemented diet (Figures 4A, B). Samples from mice in the control diet group and, to a greater extent, the I3C-supplemented diet group separated from the 7 week samples on the PC1 axis, which accounted for $50-58 \%$ of diversity in two independent experiments. Samples collected from the control diet group separated from the I3Csupplemented group on the PC2 axis, which accounted for 12$17 \%$ of diversity. Interestingly, changes in microbial diversity were established within the first week of the synthetic diet (Figure 4A).

At the phylum level (Figure 4C), the synthetic diet, regardless of supplementation, reduced the abundance of Tenericutes and increased Verrucomicrobia. The samples from mice fed the I3Csupplemented diet diverged from the control-diet group with an increase in Proteobacteria and Verrucomicrobia. In both NOD mice and patients with T1D, an increased ratio of Bacteriodetes to Firmicutes is implicated in the development of T1D (5). Consistent with dietary $\mathrm{I} 3 \mathrm{C}$ increasing insulitis, there was a significant increase in the ratio of Bacteriodetes : Firmicutes at 12 weeks of age in mice fed the I3C supplemented diet (Figure 4D). This corresponded with a positive trend $(\mathrm{p}=0.06)$ between the Bacteriodetes : Firmicutes ratio and percentage of islets with greater than $50 \%$ infiltration (Figure 4E).

\section{Transkingdom Network Analysis Identifies Bacteria in the Genera Intestinimonas, Ruminoclostridium, and Unclassified Lachnospiraceae as Key Responders to Dietary I3C}

To begin to identify which ASVs may be implicated in the immunological changes that arose following dietary I3C, we constructed a transkingdom network. Transkingdom networks have been successfully used to tease out causal interactions between mammalian host phenotypes and their colonizing microbiota (43). Here, the transkingdom network (Figure 5A) was constructed by computing correlations between host factors (hexagons) that significantly changed in mice fed the I3Csupplemented diet (Figure 3G) and ASVs identified at 12 
A

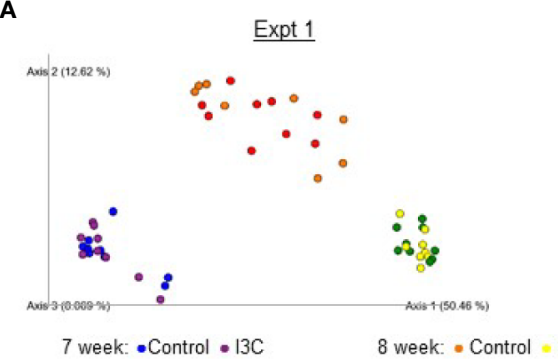

C

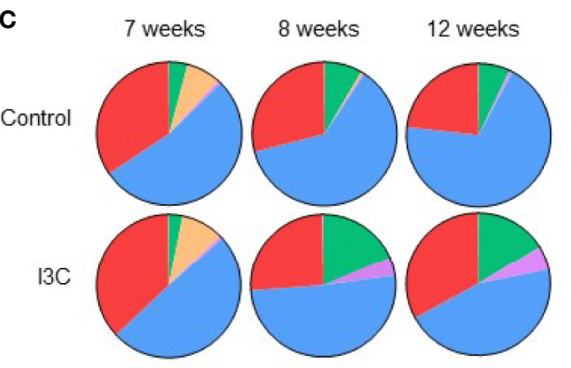

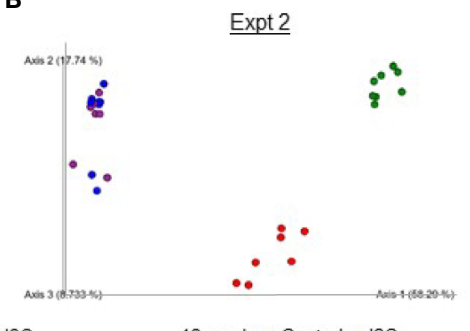

12 week: • Control • I3C

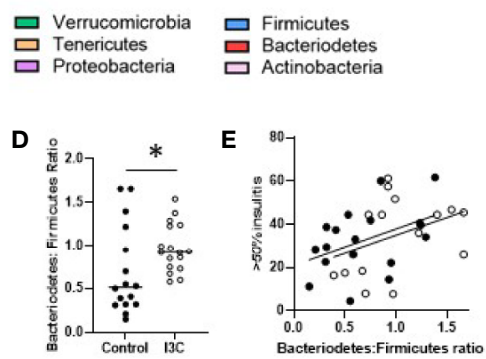

FIGURE 4 | Dietary I3C alters the gut microbial community. (A, B) Principle coordinate analysis of microbiome composition of stool samples collected prior to the dietary intervention at 7 weeks of age, after one week of the diet (8 weeks, experiment 1 only), and at the time of sacrifice at 12 weeks of age. (C) Phylum composition in mice fed a control or I3C-supplemented diet. (D) Ratio of Bacteriodetes to Firmicutes. (E) Correlation between severe insulitis and Bacteriodetes to Firmicutes ratio in control-diet (solid circles) and I3C-diet (open circles) fed mice. ${ }^{*} \mathrm{p}<0.05$.

weeks of age (circles). The network retained 108 nodes (red: increased and blue: decreased in I3C treated mice) and 221 edges, of which there were 179 positive correlations (red lines) and 42 negative correlations (blue lines).

The network was then interrogated to predict which host factors and microbes were most likely to play a role in the response to dietary I3C. We calculated two properties of the nodes, degree and bipartite betweenness centrality (BiBC) (Figure 5B); degree is the number of nodes each individual node interacts with, and measures the direct impact of one node has on other parameters in the system; bipartite betweenness centrality calculates the number of times the node lies in the shortest path connecting two groups of nodes (host parameters and ASVs). A node with a high BiBC acts as a bottleneck and thus is a regulator between processes of the system. Collectively nodes with high degrees and centrality are predicted to be critical in defining host-microbe interactions that are involved in the response to I3C. By plotting bipartite betweenness centrality and degree, we identified three ASVs, one with a high BiBC (Intestinimonas) and two with both high $\mathrm{BiBC}$ and high degree (Ruminiclostridium 9 and unclassified Lachnospiraceae) which likely regulate the abundance of other gut microbes. Intestinimonas, Ruminiclostridium, and Lachnospiraceae were decreased in $\mathrm{I} 3 \mathrm{C}$ treated mice and are members of phylum Firmicutes. On the host side, two parameters showed high $\mathrm{BiBC}$, intraepithelial $\mathrm{CD} 4^{+} \mathrm{ROR} \gamma \mathrm{t}^{+} \mathrm{Foxp} 3^{-}$cells (Figure 3D) and $\mathrm{CD} 4^{+} \mathrm{CD} 25^{+}$Foxp3-ROR $\gamma$ t(high) lamina propria cells (Figure 3E) and therefore likely influence or are influenced by microbial interactions.
The statistical importance of these five nodes was further validated by randomly generating 10,000 networks and comparing the degree and $\mathrm{BiBC}$ of these specific nodes to those in a randomly generated model. Indeed, the degrees and $\mathrm{BiBC}$ of the five nodes had a low probability of occurring randomly (Figure 5C).

While Ruminiclostridium 9, Intestinimonas, and Lachnospiraceae likely play a role in host interactions, it is unclear if their change in abundance was due to, or resulted in, changes in the intestinal immune response after I3C treatment. Therefore, we looked at the temporal abundance of these microbes (Figures 5D-F). Within one week of switching from the normal chow diet to the synthetic diet there was an increase in abundance in both Ruminiclostridium 9 and Intestinimonas regardless of $\mathrm{I} 3 \mathrm{C}$ supplementation. However, the increase in abundance of Intestinimonas was larger in the control group in comparison to the $\mathrm{I3C}$-diet group, and this pattern remained through 12 weeks of age. In contrast, the comparatively lower abundance of Ruminiclostridium 9 was not significant until 12 weeks of age. The transition from the normal diet to the synthetic diet did not significantly alter the abundance of the unclassified Lachnospiraceae ASV, however supplementation with I3C depleted the abundance of this family at both 8 and 12 weeks of age. The immediate differential abundance in Intestinimonas and Lachnospiraceae may reflect a primary response to AhR-mediated changes in the gut immune system, whereas the delayed reduced abundance in Ruminiclostridium 9 may be secondary to changes in microbial composition.

Since I3C could alter colonization with Firmicutes directly or indirectly of AhR intestinal activation, we verified if the abundance 
A

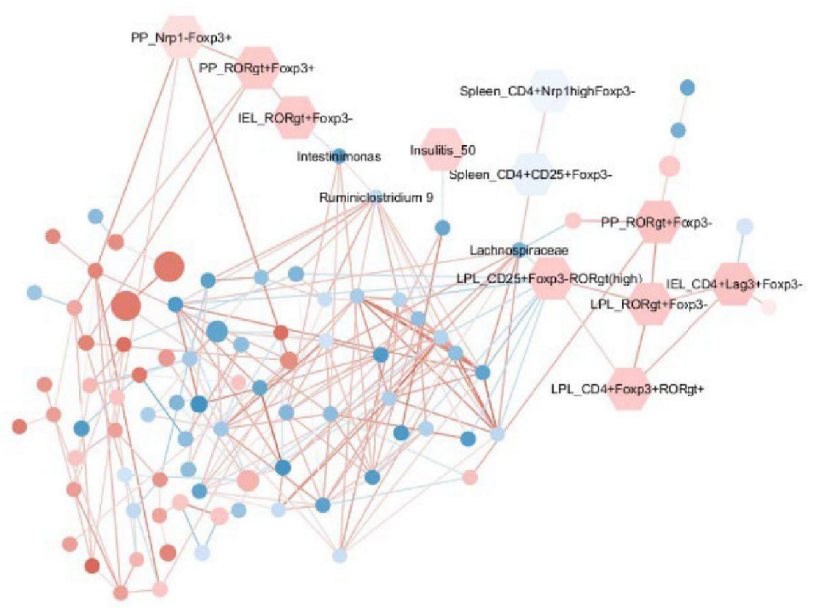

B

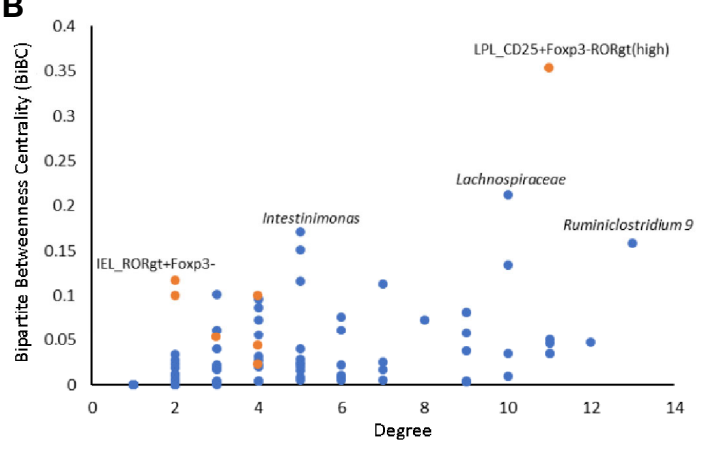

C

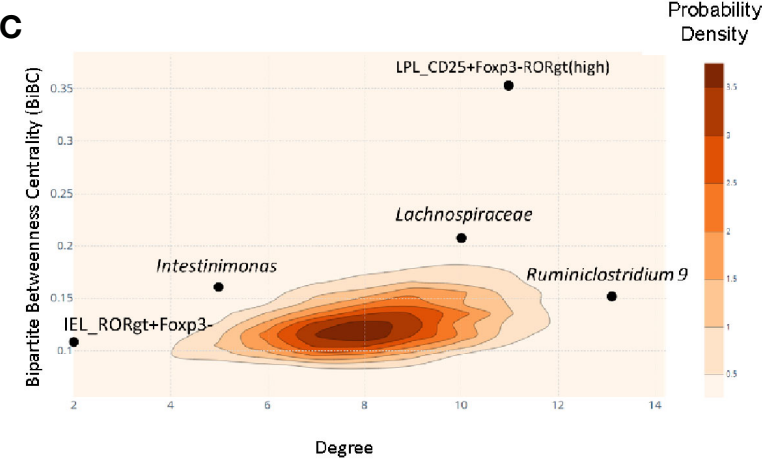

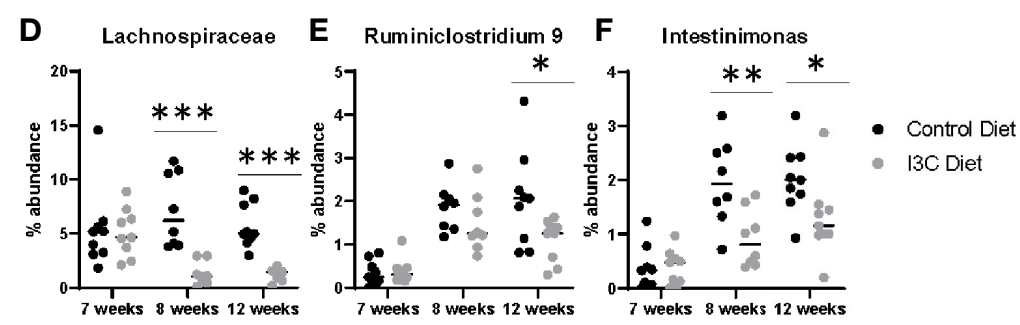
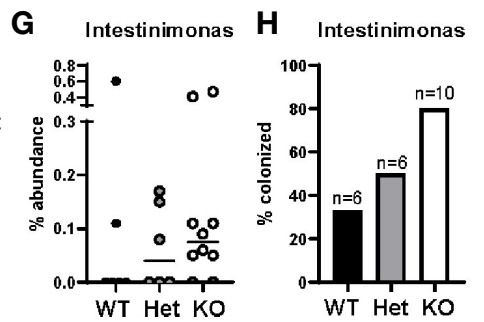

FIGURE 5 | Transkingdom network analysis identifies key host-microbe interactions. (A) Correlation network of ASVs (circles) and phenotypes (hexagons). Node color indicates the average median fold change (red is increased, blue is decreased) across all experiments. ASV node size indicates the average abundance of the ASV across both experiments. Edge color represents the average spearman correlation coefficient (red is positive, blue is negative) across all experimental groups. (B) BiBC-degree distribution of all nodes in the network, the highest of which are marked. Blue are ASVs and orange are phenotypes. (C) 2D-contour histogram of the nodes with highest $\mathrm{BiBC}$ and degree from 10,000 randomly generated networks. Darker areas indicate higher probabilities of randomly finding a node with that degree and BiBC.

(D-F) Temporal abundance of Lachnospiraceae, Ruminiclostridium 9, and Intestinimonas at 7, 8, and 12 weeks of age after treatment of I3C. (G) Abundance of Intestinimonas in NOD AhR knockout mice at 12 weeks of age. (H) Percentage of mice colonized with Intestinimonas. ${ }^{*} \mathrm{p}<0.05 ;{ }^{* \star} \mathrm{p}<0.01 ;{ }^{\star \star \star} \mathrm{p}<0.001$.

of these bacteria were affected by genetic manipulation of AhR expression. To ensure relevance of this experimental group for effect on diabetes, we used NOD AhR knockout mice at 12 weeks of age when approximately $60 \%$ of islets are infiltrated, but prior to clinical manifestations. Among the three above mentioned microbes, only Intestinimonas showed lower abundance with gene dosage. Accordingly, gut colonization by the only member of the Intestinimonas genus detected in these mice, was found in $33 \%$ of wildtype mice and $50 \%$ of heterozygous mice were colonized with Intestinimonas, $80 \%$ of their knockout littermates were colonized (Figures 5G, H). Taken together, Intestinimonas abundance is negatively regulated by $\mathrm{AhR}$ and therefore the decrease in this genus is likely to be an effect of I3C-mediated AhR signaling in the intestine.

\section{DISCUSSION}

As a molecular switch between opposing immune responses (regulatory and inflammatory), activation of the AhR can either 
protect from or promote the development of immune-mediated diseases. Understanding how immune modulation is impacted by different AhR ligands, their routes of administration, and their biodistribution is important for determining if AhR ligands may act as an environmental risk factor for T1D development, and/or how AhR can be exploited as a target for T1D treatment. In the current study we found that gut-localized AhR activation by I3C increased Th17 cells and exacerbated T1D in NOD mice, in contrast to our previous findings that systemic AhR activation by Cl-BBQ and TCDD reduced Th17 cells and prevented the development of T1D $(10,44)$. These results suggest that the site of $\mathrm{AhR}$ activation influences the outcome of $\mathrm{CD} 4^{+} \mathrm{T}$ cell differentiation.

These contrasting effects on $\mathrm{CD} 4^{+} \mathrm{T}$ cell differentiation do not appear to be a consequence of the extent of AhR activation. Using an alloresponse model, we previously found that if you can normalize the extent $\mathrm{AhR}$ activation, $\mathrm{CD} 4^{+} \mathrm{T}$ cell differentiation follows a predictable pattern. Low levels of AhR activation promote Th17 cells and high levels promote $\operatorname{Tr} 1$ cell differentiation (15). While dietary I3C only led to limited AhR activation in the periphery in comparison to $\mathrm{Cl}-\mathrm{BBQ}$, the opposite occurred in the small intestine; dietary I3C induced 2,000-fold higher induction of Cyplal than Cl-BBQ in the ileum. By strongly activating Cyp1a1 in the small intestine, it is possible that the AhR activating catabolites of I3C (e.g. ICZ), were rapidly metabolized, inducing feedback control that prevented systemic distribution (45). Despite inducing an environment where I3C led to strong local AhR activation, Th17 cells were increased in the intestine. The organ site of AhR activation (intestine vs periphery) with different microenvironments, cell composition, and architecture may be a missing variable controlling the outcome of AhR-signaling in $\mathrm{CD} 4^{+} \mathrm{T}$ cells.

While systemic Th17 cells are implicated in the development of T1D (17-19, 41), it is less clear how intestinal Th17 cells impact disease development. Their role during T1D is further complicated by studies in which intestinal Th17 are ascribed both proinflammatory and homeostatic properties. These differing functionalities may be dependent on how intestinal Th17 cells develop in concert with the microbiome. Th17 cells appear in mice at the time of wean corresponding to cecum development and establishment of the microbiota (46). It is conceivable, therefore, that initiating dietary $\mathrm{I} 3 \mathrm{C}$ at the time of wean, rather than at 7 weeks of age, could negate the I3C-mediated increase in Th17 cells. One study in germ-free C57BL/6 mice, showed that Th17 cells do not develop in the small intestine lamina propria, and fecal transfer from SPF mice restored IL-17 production. In contrast, a study found that germ-free NOD mice have both increased Th17 cells and Th1 cells resulting in exacerbated insulitis (47). By comparing diabetes incidence in multiple housing facilities, it was found that colonization with segmented-filamentous bacteria (SFB) could be used to stratify an increase in Th17 cells and reduced T1D incidence (48). The protective effect of SFB requires a diverse microbiota, as monocolonization with SFB does not protect from T1D (49). Of note, AhR regulates SFB colonization (50), although SFB was not present in our mouse colony during the current study. A recent paper by Omenetti et al. poses that these contradictory findings are due to the presence of two different types of resident Th17 cells, homeostatic (which can be generated from SFB) and inflammatory (which can be generated from $C$. rodentium) (51). The homeostatic Th17 cells play a role in barrier protection and have a limited metabolic program whereas the inflammatory Th17 cells produce IFN $\gamma$ and are able to migrate into the periphery. Therefore, the functionality of Th17 cells depend on their interaction with specific microbes, and may explain the conflicting literature on gut Th17 cells and T1D pathogenesis.

Intestinal Th17 cell differentiation following AhR activation may proceed through both direct $\mathrm{CD} 4^{+} \mathrm{T}$ cell signaling and indirectly through AhR-mediated modulation of the gut microbiome. AhR is highly expressed in Th17 cells. Under Th17 differentiating conditions AhR activation, in combination with Stat3, activate Aiolos, inhibiting IL-2, and promoting Th17 differentiation (52). Th17 differentiating conditions are present in the intestine originating from microbial metabolites (including AhR ligands), microbe-mediated PRR activation, and microbial-secreted ATP, where binding to purinergic receptors expressed on LP dendritic cells along with TLR activation increases Th17-skewing IL-6 and TGF- $\beta$ production $(53,54)$ Microbial activation of the NLRP3/IL$1 \beta$ signaling pathway can also induce intestinal Th17 polarization (55), although, interestingly, AhR signaling has been shown to negatively regulate inflammasome activation (56). Thus, it likely that a combination of (and sometimes opposing) Th17-promoting factors could be implicated in the dietary I3C-mediated increase in intestinal $\mathrm{CD}^{+}{ }^{+} \mathrm{ROR} \gamma \mathrm{t}^{+}$Foxp3- cells in NOD mice.

In our study, dietary I3C strongly skewed the gut microbial community and increased the Bacteriodetes : Firmicutes ratio. In mouse studies, this increased ratio is positively correlated with T1D, and prediabetic children have an increased abundance of Bacteriodetes $(5,57,58)$. However, the utility of using this ratio as predictive for T1D development is inconsistent, and is further complicated by the site of bacteria collected for analysis. Fecal bacteria (as measured in this study), have higher abundance of Bacteriodetes compared to cecal content (59). Using a transkingdom network analysis, we identified three genera belonging to the Firmicutes phylum that are predicted to be involved in the immune modulation by dietary I3C, Intestinimonas, Ruminiclostrdium 9 and unclassified Lachnospiraceae. Based on their network properties and follow up studies in AhR knockout mice, we predict that Intestinimonas is directly regulated by AhR activation, whereas Ruminiclostridium 9 and Lachnospiraceae may be altered indirectly in response to AhR-host-microbe interactions. All three of the bacteria identified as key contributors to the network are butyrate producers (60-62); butyrate helps promote intestinal integrity and increases Tregs. NOD mice fed a diet that increases butyrate production by the gut microbiota had decreased insulitis (63). Butyrate-mediated protection from T1D corresponded with tolerized DCs and an increase in Tregs in the colon but not in the pancreatic lymph node. The protective role of butyrate is consistent with the reduction in butyrate-producing bacteria in mice given dietary I3C. Interestingly, a recent study showed that i.p. injection of I3C resulted in an increase in butyrate-producing gut bacteria in a 
C57BL/6 mouse model of TNBS-induced colitis (37). I3C-mediated resolution of disease and increase in butyrate-producing microbes were dependent on the induction of IL-22. IL-22 has been reported as one of the main mechanisms by which $\mathrm{AhR}$ can regulate the gut microbiome and immune homeostasis (37, 50, 64-66). However, in our study with NOD mice, I3C did not alter IL-22 expression in the small intestine when measured by qPCR or by flow cytometry (data not shown).

Additional differences between the AhR response in NOD and C57BL/6 mice are highlighted when comparing our study with findings in a model of oral tolerance. In NOD mice, TCDD and ClBBQ (when administered at a dose-rate to match the activation of AhR by TCDD) prevent insulitis (10), and I3C exacerbates disease. Conversely, in the oral tolerance model, I3C promotes oral tolerance and TCDD breaks oral tolerance $(26,67)$. Interestingly, in these C57BL/6 mice, TCDD induced an order of magnitude higher Cypla1 in the small intestine compared to $\mathrm{I} 3 \mathrm{C}$, and led to a small, but significant increase in Th17 cells in the mesenteric lymph nodes. Thus, an important unresolved question is why does I3C lead to immune regulation, expand intestinal Tregs and promote IL-22 production in other C57BL/6 mouse models, when the opposite occurs in NOD mice. One clue might come from a study that looked at the impact of AhR allele on the ability of dietary broccoli to suppress colitis. Using C57BL/6 mice that express either the $\mathrm{AhR}^{\mathrm{b}}$ allele (wildtype, high affinity) or congenic C57BL/6 mice with the reduced sensitivity $A_{h R}{ }^{d}$ allele (68), it was found that AhR sensitivity determined disease outcome. In the wild type C57BL/6 mice, dietary broccoli attenuated colitis and reduced Th17 cells, similar to the effect of $\mathrm{I} 3 \mathrm{C}$ in colitis models. In contrast, $\mathrm{AhR}^{\mathrm{d}}$ mice fed the broccoli-supplemented diet had a 1.6-fold increase in splenic Th17 cells. Since NOD mice express the lower affinity $A_{h R^{d}}$ allele, (12), this genetic difference may represent an additional factor controlling AhR-mediated $\mathrm{CD}^{+} \mathrm{T}$ cell modulation. The differences in allele sensitivity may also explain contradictory findings in regard to IL-22. In NOD mice, pancreatic islets have defective Il22 expression compared to BALB/c $\left(\mathrm{AhR}^{\mathrm{b}}\right)$ mice despite having similar concentrations of AhR ligands in their feces and serum (69). Humans express an AhR with similar ligand sensitivity to the murine $A h R^{d}$ allele, thus the $A h R^{d}$ model may be more appropriate for predicting immune modulation in humans in response to dietary AhR ligands.

The restriction of AhR activation to the intestine following dietary I3C and the promotion of insulitis was unexpected. However, these results provided new insights on the role of AhR allele sensitivity, intestinal Th17 cells and gut microbial composition during the development of T1D. Additionally, these findings highlight potential risks associated with dietary I3C supplementation.

\section{REFERENCES}

1. Verdu EF, Danska JS. Common ground: shared risk factors for type 1 diabetes and celiacdisease. Nat Immunol. (2018) 19(7):685-95. doi: 10.1038/s41590018-0130-2

2. Rewers M, Ludvigsson J. Environmental risk factors for type 1 diabetes. Lancet (2016) 387(10035):2340-8. doi: 10.1016/S0140-6736(16)30507-4

3. Virtanen SM. Dietary factors in the development of type 1 diabetes. Pediatr Diabetes (2016) 17(Suppl 22):49-55. doi: 10.1111/pedi.12341

\section{DATA AVAILABILITY STATEMENT}

The data presented in the study are deposited in the NCBI Sequence Read Archive, accession number PRJNA679964.

\section{ETHICS STATEMENT}

The animal study was reviewed and approved by the Institutional Animal Care and Use Committee at Oregon State University.

\section{AUTHOR CONTRIBUTIONS}

HK, NN, JP, and AE performed the experiments. NS, AM, and AE contributed to the conception and design of the study. NM, $\mathrm{AM}$, and $\mathrm{AE}$ analyzed the data. AE, NK, SK, and NS provided funding for the project. $\mathrm{AE}, \mathrm{NN}$, and $\mathrm{NK}$ wrote the manuscript. All authors contributed to the article and approved the submitted version.

\section{FUNDING}

This work was supported by the NIDDK (grant numbers 1K99DK117509-01 and 4 R00 DK117509-03 awarded to AE, R01 DK103761 awarded to NS) and NIEHS (grant number 5R01ES016651 awarded to NK and SK) and startup resources from UC Davis.

\section{ACKNOWLEDGMENTS}

The authors would like to thank Matthew Rolston and the HostMicrobe Systems Biology Core Facility at UC Davis for library preparation and sequencing, Rachel Shan for assistance with sample collection and isolation, David Williams for helpful advice regarding dietary I3C, and Hannah You for fecal DNA isolation training.

\section{SUPPLEMENTARY MATERIAL}

The Supplementary Material for this article can be found online at: https://www.frontiersin.org/articles/10.3389/fimmu.2020. 606441/full\#supplementary-material

4. Bosi E, Molteni L, Radaelli MG, Folini L, Fermo I, Bazzigaluppi E, et al. Increased intestinal permeability precedes clinical onset of type 1 diabetes. Diabetologia (2006) 49(12):2824-7. doi: 10.1007/s00125-006-0465-3

5. Gülden E, Wong FS, Wen L. The gut microbiota and Type 1 Diabetes. Clin Immunol (2015) 159(2):143-53. doi: 10.1016/j.clim.2015.05.013

6. Kostic AD, Gevers D, Siljander H, Vatanen T, Hyötyläinen T, Hämäläinen AM, et al. The dynamics of the human infant gut microbiome in development and in progression toward type 1 diabetes. Cell Host Microbe (2015) 17 (2):260-73. doi: 10.1016/j.chom.2015.01.001 
7. Yap YA, Mariño E. An Insight Into the Intestinal Web of Mucosal Immunity, Microbiota, and Diet in Inflammation. Front Immunol (2018) 9:2617:2617. doi: 10.3389/fimmu.2018.02617

8. Hubbard TD, Murray IA, Perdew GH. Indole and Tryptophan Metabolism: Endogenous and Dietary Routes to Ah Receptor Activation. Drug Metab Dispos (2015) 43(10):1522-35. doi: 10.1124/dmd.115.064246

9. Rothhammer V, Quintana FJ. The aryl hydrocarbon receptor: an environmental sensor integrating immune responses in health and disease. Nat Rev Immunol (2019) 19(3):184-97. doi: 10.1038/s41577-019-0125-8

10. Ehrlich AK, Pennington JM, Wang X, Rohlman D, Punj S, Löhr CV, et al. Activation of the Aryl Hydrocarbon Receptor by 10-Cl-BBQ Prevents Insulitis and Effector T Cell Development Independently of Foxp3+ Regulatory T Cells in Nonobese Diabetic Mice. J Immunol (2016) 196(1):264-73. doi: 10.4049/ jimmunol.1501789

11. Hauben E, Gregori S, Draghici E, Migliavacca B, Olivieri S, Woisetschläger M, et al. Activation of the aryl hydrocarbon receptor promotes allograft-specific tolerance through direct and dendritic cell-mediated effects on regulatory $\mathrm{T}$ cells. Blood (2008) 112(4):1214-22. doi: 10.1182/blood-2007-08-109843

12. Kerkvliet NII, Steppan LB, Vorachek W, Oda S, Farrer D, Wong CP, et al. Activation of aryl hydrocarbon receptor by TCDD prevents diabetes in NOD mice and increases Foxp3+ T cells in pancreatic lymph nodes. Immunotherapy (2009) 1(4):539-47. doi: 10.2217/imt.09.24

13. Yeste A, Takenaka MC, Mascanfroni ID, Nadeau M, Kenison JE, Patel B, et al. Tolerogenic nanoparticles inhibit $\mathrm{T}$ cell-mediated autoimmunity through SOCS2. Sci Signal (2016) 9(433):ra61. doi: 10.1126/scisignal.aad0612

14. Yue T, Sun F, Yang C, Wang F, Luo J, Yang P, et al. The AHR Signaling Attenuates Autoimmune Responses During the Development of Type 1 Diabetes. Front Immunol (2020) 11:1510:1510. doi: 10.3389/fimmu. 2020.01510

15. Ehrlich AK, Pennington JM, Bisson WH, Kolluri SK, Kerkvliet NII. TCDD, FICZ, and Other High Affinity AhR Ligands Dose-Dependently Determine the Fate of CD4+ T Cell Differentiation. Toxicol Sci (2018) 161(2):310-20. doi: $10.1093 /$ toxsci/kfx215

16. Abdel-Moneim A, Bakery HH, Allam G. The potential pathogenic role of IL17/Th17 cells in both type 1 and type 2 diabetes mellitus. BioMed Pharmacother (2018) 101:287-92. doi: 10.1016/j.biopha.2018.02.103

17. Bending D, De la Peña H, Veldhoen M, Phillips JM, Uyttenhove C, Stockinger B, et al. Highly purified Th17 cells from BDC2.5NOD mice convert into Th1-like cells in NOD/SCID recipient mice. J Clin Invest (2009) 119(3):565-72. doi: $10.1172 /$ JCI37865

18. Emamaullee JA, Davis J, Merani S, Toso C, Elliott JF, Thiesen A, et al. Inhibition of Th17 cells regulates autoimmune diabetes in NOD mice. Diabetes (2009) 58(6):1302-11. doi: 10.2337/db08-1113

19. Kuriya G, Uchida T, Akazawa S, Kobayashi M, Nakamura K, Satoh T, et al. Double deficiency in IL-17 and IFN- $\gamma$ signalling significantly suppresses the development of diabetes in the NOD mouse. Diabetologia (2013) 56(8):177380. doi: 10.1007/s00125-013-2935-8

20. Higdon JV, Delage B, Williams DE, Dashwood RH. Cruciferous vegetables and human cancer risk: epidemiologic evidence and mechanistic basis. Pharmacol Res (2007) 55(3):224-36. doi: 10.1016/j.phrs.2007.01.009

21. N. T. Program. NTP Technical Report on the Toxicology Studies of Indole-3carbinol(CASRN 700-06-1) in F344/N Rats and B6C3F1/N Mice and Toxicology and Carcinogenesis Studies ofIndole-3-carbinol in Harlan Sprague Dawley Rats and B6C3F1/N Mice (Gavage Studies). In: Technical Report 584. Research Triangle Park, North Carolina, US: Public Health Service U.S. Department of Health and Human Services (2017).

22. Li Y, Innocentin S, Withers DR, Roberts NA, Gallagher AR, Grigorieva EF, et al. Exogenous stimuli maintain intraepithelial lymphocytes via aryl hydrocarbon receptor activation. Cell (2011) 147(3):629-40. doi: 10.1016/ j.cell.2011.09.025

23. Schanz O, Chijiiwa R, Cengiz SC, Majlesain Y, Weighardt H, Takeyama H, et al. Dietary AhR Ligands Regulate AhRR Expression in Intestinal ImmuneCells and Intestinal Microbiota Composition. Int J Mol Sci (2020) 21(9):3189. doi: 10.3390/ijms21093189

24. Wu Y, He Q, Yu L, Pham Q, Cheung L, Kim YS, et al. Indole-3-Carbinol Inhibits Citrobacterrodentium Infection through Multiple Pathways Including Reduction of Bacterial Adhesion and Enhancement of Cytotoxic T Cell Activity. Nutrients (2020) 12(4):917. doi: 10.3390/nu12040917
25. Julliard W, De Wolfe TJ, Fechner JH, Safdar N, Agni R, Mezrich JD. Amelioration of Clostridium difficile Infection in Mice by Dietary Supplementation With Indole-3-carbinol. Ann Surg (2017) 265(6):1183-91. doi: $10.1097 /$ SLA.0000000000001830

26. Hammerschmidt-Kamper C, Biljes D, Merches K, Steiner I, Daldrup T, BolSchoenmakers $\mathrm{M}$, et al. Indole-3-carbinol, a plant nutrient and AhR-Ligand precursor, supports oral tolerance against OVA and improves peanut allergy symptoms in mice. PloS One (2017) 12(6):e0180321. doi: 10.1371/ journal.pone. 0180321

27. Brawner KM, Yeramilli VA, Duck LW, Van Der Pol W, Smythies LE, Morrow $\mathrm{CD}$, et al. Depletion of dietary aryl hydrocarbon receptor ligands alters microbiota composition and function. Sci Rep (2019) 9(1):14724. doi: 10.1038/s41598-019-51194-w

28. Dedrick S, Sundaresh B, Huang Q, Brady C, Yoo T, Cronin C, et al. The Role of Gut Microbiota and Environmental Factors in Type 1 Diabetes Pathogenesis. Front Endocrinol (Lausanne) (2020) 11:78:78. doi: 10.3389/ fendo.2020.00078

29. Goodyear AW, Kumar A, Dow S, Ryan EP. Optimization of murine small intestine leukocyte isolation for global immune phenotype analysis. J Immunol Methods (2014) 405:97-108. doi: 10.1016/j.jim.2014.01.014

30. Bolyen E, Rideout JR, Dillon MR, Bokulich NA, Abnet CC, Al-Ghalith GA, et al. Reproducible, interactive, scalable and extensible microbiome datascience using QIIME 2. Nat Biotechnol (2019) 37(8):852-7. doi: 10. 1038/s41587-019-0209-9

31. Callahan BJ, McMurdie PJ, Rosen MJ, Han AW, Johnson AJ, Holmes SP. DADA2: High-resolution sample inference from Illumina amplicon data. Nat Methods (2016) 13(7):581-3. doi: 10.1038/nmeth.3869

32. Dong X, Yambartsev A, Ramsey SA, Thomas LD, Shulzhenko N, Morgun A. Reverse enGENEering of Regulatory Networks from Big Data: A Roadmap for Biologists. Bioinform Biol Insights (2015) 9:61-74. doi: 10.4137/ BBI.S12467

33. Rodrigues RR, Shulzhenko N, Morgun A. Transkingdom Networks: A Systems Biology Approach to Identify Causal Members of Host-Microbiota Interactions. Methods Mol Biol (2018) 1849:227-42. doi: 10.1007/978-1-49398728-3_15

34. Yambartsev A, Perlin MA, Kovchegov Y, Shulzhenko N, Mine KL, Dong X, et al. Unexpected links reflect the noise in networks. Biol Direct (2016) 11 (1):52. doi: 10.1186/s13062-016-0155-0

35. Balduzzi S, Rücker G, Schwarzer G. How to perform a meta-analysis with R: a practical tutorial. Evid Based Ment Health (2019) 22(4):153-60. doi: 10.1136/ ebmental-2019-300117

36. Shannon P, Markiel A, Ozier O, Baliga NS, Wang JT, Ramage D, et al. Cytoscape: a software environment for integrated models of biomolecular interaction networks. Genome Res (2003) 13(11):2498-504. doi: 10.1101/ gr.1239303

37. Busbee PB, Menzel L, Alrafas HR, Dopkins N, Becker W, Miranda K, et al. Indole-3-carbinol prevents colitis and associated microbialdysbiosis in an IL22-dependent manner. JCI Insight(2020) 5(1):e127551. doi: 10.1172/ jci.insight. 127551

38. Okey AB, Vella LM, Harper PA. Detection and characterization of a low affinity form of cytosolic Ah receptor in livers of mice nonresponsive to induction of cytochrome P1-450 by 3-methylcholanthrene. Mol Pharmacol (1989) 35(6):823-30.

39. Nukaya M, Moran S, Bradfield CA. The role of the dioxin-responsive element cluster between the Cypla1 and Cyp1a2 loci in aryl hydrocarbon receptor biology. Proc Natl Acad Sci U S A (2009) 106(12):4923-8. doi: 10.1073/ pnas.0809613106

40. Bluestone JA, Buckner JH, Fitch M, Gitelman SE, Gupta S, Hellerstein MK, et al. Type 1 diabetes immunotherapy using polyclonal regulatory T cells. Sci Transl Med (2015) 7(315):315ra189. doi: 10.1126/scitranslmed.aad4134

41. Solt LA, Banerjee S, Campbell S, Kamenecka TM, Burris TP. ROR inverse agonist suppresses insulitis and prevents hyperglycemia in a mouse model of type 1 diabetes. Endocrinology (2015) 156(3):869-81. doi: 10.1210/en.2014-1677

42. Spence A, Tang Q. Restoring Regulatory T Cells in Type 1 Diabetes. Curr Diabetes Rep (2016) 16(11):110. doi: 10.1007/s11892-016-0807-6

43. Greer R, Dong X, Morgun A, Shulzhenko N. Investigating a holobiont: Microbiota perturbations and transkingdom networks. Gut Microbes (2016) 7(2):126-35. doi: 10.1080/19490976.2015.1128625 
44. Punj S, Kopparapu P, Jang HS, Phillips JL, Pennington J, Rohlman D, et al. Benzimidazoisoquinolines: a new class of rapidly metabolized aryl hydrocarbon receptor (AhR) ligands that induce AhR-dependent Tregs and prevent murine graft-versus-host disease. PLoS One (2014) 9(2):e88726. doi: 10.1371/journal.pone.0088726

45. Schiering C, Wincent E, Metidji A, Iseppon A, Li Y, Potocnik AJ, et al. Feedback control of AHR signalling regulates intestinal immunity. Nature (2017) 542(7640):242-5. doi: 10.1038/nature21080

46. Ivanov III, Frutos REL, Manel N, Yoshinaga K, Rifkin DB, Sartor RB, et al. Specific microbiota direct the differentiation of IL-17-producing T-helper cells in the mucosa of the small intestine. Cell Host Microbe (2008) 4(4):337-49. doi: 10.1016/j.chom.2008.09.009

47. Alam C, Bittoun E, Bhagwat D, Valkonen S, Saari A, Jaakkola U, et al. Effects of a germ-free environment on gut immune regulation and diabetes progression in non-obese diabetic (NOD) mice. Diabetologia (2011) 54 (6):1398-406. doi: 10.1007/s00125-011-2097-5

48. Kriegel MA, Sefik E, Hill JA, Wu HJ, Benoist C, Mathis D. Naturally transmitted segmented filamentous bacteria segregate with diabetes protection in nonobese diabetic mice. Proc Natl Acad Sci U S A (2011) 108 (28):11548-53. doi: 10.1073/pnas.1108924108

49. Yurkovetskiy L, Burrows M, Khan AA, Graham L, Volchkov P, Becker L, et al. Gender bias in autoimmunity is influenced by microbiota. Immunity (2013) 39(2):400-12. doi: 10.1016/j.immuni.2013.08.013

50. Qiu J, Guo X, Chen ZM, He L, Sonnenberg GF, Artis D, et al. Group 3 innate lymphoid cells inhibit T-cell-mediated intestinal inflammation through aryl hydrocarbon receptor signaling and regulation of microflora. Immunity (2013) 39(2):386-99. doi: 10.1016/j.immuni.2013.08.002

51. Omenetti S, Bussi C, Metidji A, Iseppon A, Lee S, Tolaini M, et al. The Intestine Harbors Functionally Distinct Homeostatic Tissue-Resident and Inflammatory Th17 Cells. Immunity (2019) 51(1):77-89.e6. doi: 10.1016/ j.immuni.2019.05.004

52. Quintana FJ, Jin H, Burns EJ, Nadeau M, Yeste A, Kumar D, et al. Aiolos promotes TH17 differentiation by directly silencing Il2 expression. Nat Immunol (2012) 13(8):770-7. doi: 10.1038/ni.2363

53. Chewning JH, Weaver CT. Development and survival of Th17 cells within the intestines: the influence of microbiome- and diet-derived signals. J Immunol (2014) 193(10):4769-77. doi: 10.4049/jimmunol.1401835

54. Pandiyan P, Bhaskaran N, Zou M, Schneider E, Jayaraman S, Huehn J. Microbiome Dependent Regulation of T. Front Immunol (2019) 10:426:426. doi: 10.3389/fimmu.2019.00426

55. Mak'Anyengo R, Duewell P, Reichl C, Hörth C, Lehr HA, Fischer S, et al. Nlrp3-dependent IL-1 $\beta$ inhibits CD103+ dendritic cell differentiation in the gut. JCI Insight (2018) 3(5):e96322. doi: 10.1172/jci.insight.96322

56. Ngui IQH, Perera AP, Eri R. Does NLRP3 Inflammasome and Aryl Hydrocarbon Receptor Play an Interlinked Role in Bowel Inflammation and Colitis-Associated Colorectal Cancer? Molecules (2020) 25(10):2427. doi: $10.3390 /$ molecules 25102427

57. Brown CT, Davis-Richardson AG, Giongo A, Gano KA, Crabb DB, Mukherjee N, et al. Gut microbiome metagenomics analysis suggests a functional model for the development of autoimmunity for type 1 diabetes. PloS One (2011) 6(10):e25792. doi: 10.1371/journal.pone.0025792

58. Wolf KJ, Daft JG, Tanner SM, Hartmann R, Khafipour E, Lorenz RG. Consumption of acidic water alters the gut microbiome and decreases the risk of diabetes in NOD mice. J Histochem Cytochem (2014) 62(4):237-50. doi: $10.1369 / 0022155413519650$
59. Tanca A, Manghina V, Fraumene C, Palomba A, Abbondio M, Deligios M, et al. Metaproteogenomics Reveals Taxonomic and Functional Changes between Cecal and Fecal Microbiota in Mouse. Front Microbiol (2017) 8:391:391. doi: 10.3389/fmicb.2017.00391

60. Afouda P, Durand GA, Lagier JC, Labas N, Cadoret F, Armstrong N, et al. Noncontiguous finished genome sequence and description of Intestinimonas massiliensis sp. nov strain GD2. Microbiologyopen (2019) 8(1):e00621. doi: $10.1002 / \mathrm{mbo} 3.621$

61. Vacca M, Celano G, Calabrese FM, Portincasa P, Gobbetti M, De Angelis M. The Controversial Role of Human Gut Lachnospiraceae. Microorganisms (2020) 8(4):573. doi: 10.3390/microorganisms8040573

62. Vital M, Howe AC, Tiedje JM. Revealing the bacterial butyrate synthesis pathways by analyzing (meta)genomic data. mBio (2014) 5(2):e00889. doi: $10.1128 / \mathrm{mBio} .00889-14$

63. Mariño E, Richards JL, McLeod KH, Stanley D, Yap YA, Knight J, et al. Gut microbial metabolites limit the frequency of autoimmune $\mathrm{T}$ cells and protect against type 1 diabetes. Nat Immunol (2017) 18(5):552-62. doi: 10.1038/ ni.3713

64. Yeste A, Mascanfroni ID, Nadeau M, Burns EJ, Tukpah AM, Santiago A, et al. IL-21 induces IL-22 production in CD4+ T cells. Nat Commun (2014) 5:3753. doi: $10.1038 /$ ncomms 4753

65. Zelante T, Innitti RG, Cunha C, De Luca A, Giovannini G, Pieraccini G, et al. Tryptophan catabolites from microbiota engage aryl hydrocarbon receptor and balance mucosal reactivity via interleukin-22. Immunity (2013) 39 (2):372-85. doi: 10.1016/j.immuni.2013.08.003

66. Monteleone I, Rizzo A, Sarra M, Sica G, Sileri P, Biancone L, et al. Aryl hydrocarbon receptor-induced signals up-regulate IL-22 production and inhibit inflammation in the gastrointestinal tract. Gastroenterology (2011) 141(1):237-48, 248.el. doi: 10.1053/j.gastro.2011.04.007

67. Chmill S, Kadow S, Winter M, Weighardt H, Esser C. 2,3,7,8Tetrachlorodibenzo-p-dioxin impairs stable establishment of oral tolerance in mice. Toxicol Sci (2010) 118(1):98-107. doi: 10.1093/toxsci/ kfq232

68. Hubbard TD, Murray IA, Nichols RG, Cassel K, Podolsky M, Kuzu G, et al. Dietary Broccoli Impacts Microbial Community Structure and Attenuates Chemically Induced Colitis in Mice in an Ah receptor dependent manner. J Funct Foods (2017) 37:685-98. doi: 10.1016/ j.jff.2017.08.038

69. Miani M, Le Naour J, Waeckel-Enée E, Verma SC, Straube M, Emond P, et al. Gut Microbiota-Stimulated Innate Lymphoid Cells Support $\beta$ Defensin 14 Expression in Pancreatic Endocrine Cells, Preventing Autoimmune Diabetes. Cell Metab (2018) 28(4):557-572.e6. doi: 10.1016/ j.cmet.2018.06.012

Conflict of Interest: The authors declare that the research was conducted in the absence of any commercial or financial relationships that could be construed as a potential conflict of interest.

Copyright (c) 2021 Kahalehili, Newman, Pennington, Kolluri, Kerkvliet, Shulzhenko, Morgun and Ehrlich. This is an open-access article distributed under the terms of the Creative Commons Attribution License (CC BY). The use, distribution or reproduction in other forums is permitted, provided the original author(s) and the copyright owner(s) are credited and that the original publication in this journal is cited, in accordance with accepted academic practice. No use, distribution or reproduction is permitted which does not comply with these terms. 